\title{
Steering Toward a Quantum Advantage
}

\author{
New experiments make clear how quantum effects-in the form of \\ quantum correlations-can make an engine perform better than classical \\ limits.
}

By Michael Schirber

A n engine manipulated by a "demon" can generate more work than would be expected from basic thermodynamics, but whether a quantum demon can outperform a classical demon is still an open question. A new experimental study shows that correlations-specifically a type of correlation called quantum steering-can allow more work to be extracted from an atomic-scale engine than classically allowed [1].

A demon-like the one imagined by the physicist Leo Szilárd in 1929-can measure particles inside an engine and then use that information to configure the engine so that more work is generated. Despite appearances, this enhanced output is not a violation of thermodynamic limits, as the demon's information gathering factors into the entropy budget (see Viewpoint: Maxwell's Demon Meets Nonequilibrium Quantum Thermodynamics).
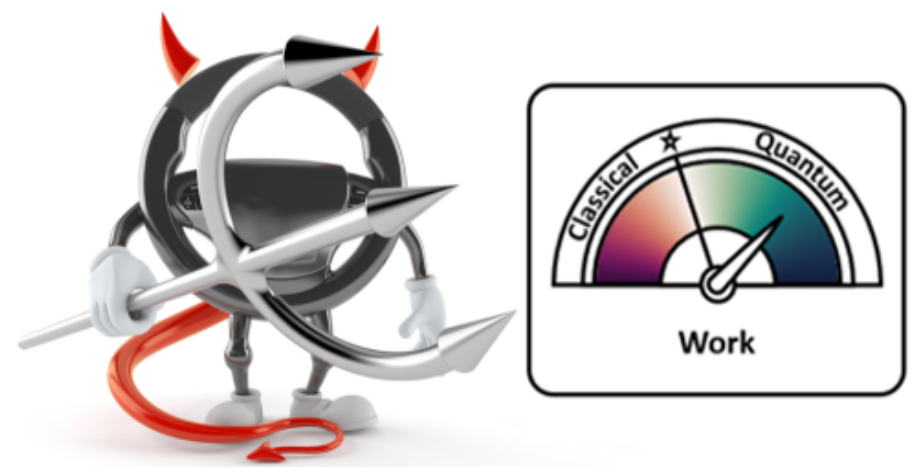

Credit: J. Du; Talaj/stock.adobe.com
Recent work has looked at quantum Szilárd engines, but their advantage over classical engines has been unclear, in part because of ambiguity over what constitutes "quantumness" in thermodynamic systems. Now, Jiangfeng Du from the University of Science and Technology of China and colleagues have devised an engine whose quantumness is clearly identified as coming from quantum correlations. The team's engine is a diamond defect, called a nitrogen-vacancy center, whose nuclear and electronic spins play the role of the working medium and thermal bath, respectively. The researchers initialize the system with steering-type correlations between the medium and bath. They show that a demon can measure the bath and use that information to optimize work-extracting operations on the medium. They find that the work from a correlated (quantum) engine exceeds that of an uncorrelated (classical) engine. The researchers expect that quantum correlations will be a central factor in other quantum heat engines.

Michael Schirber is a Corresponding Editor for Physics based in Lyon, France.

\section{REFERENCES}

1. W. Ji et al., "Spin quantum heat engine quantified by quantum steering," Phys. Rev. Lett. 128, 090602 (2022). 